example of self-monitoring clinical audit, increasingly considered an important part of continuing professional education (Batstone, 1990). In addition, a completed log book is a valuable aide-memoire when compiling a curriculum vitae.

As demands during training increase, an important role for the log-book may be to aid the review of prior experience and career plans in order to prioritise future components of training. As suggested by Cole \& Scott (1991), completing such a document would encourage the development of time-management skills in preparation for a consultant post, which is particularly important at senior registrar level.

For trainers and the College, log-books can only improve the monitoring and assessment of training schemes, and so further benefit trainees.

\section{Risks associated with log-books}

The most obvious risk is that log-books will not be kept up to date, particularly if they are seen by trainees as an imposition. Child psychiatrists are facing many changes; audit, reforms within the Health Service and the Children Act, and so any innovations (no matter how small) will need to be eased into existence. Durability will be enhanced by an open dialogue between all parties and by including some flexibility, in order to develop a system that is seen as valuable to trainees (Glaser \& Backer, 1980). Some trainees have expressed anxiety that valuable discussions in supervision might be reduced to simply handing in the log-book for review!

\section{Conclusion}

We welcome the suggestion of a system of selfevaluation and monitoring during higher training in CAP, and would like to encourage ongoing discussion about how this could be achieved.

\section{References}

BATSTONE, G. F. (1990) Educational aspects of medical audit. British Medical Journal, 301, 326-328.

Cole, A. \& SCotT, J. (1991) A study of the use of log books in the training of psychiatrists. Psychiatric Bulletin, 15, 214-216.

GLASER, E. M. \& BACKER, T. E. (1980) Durability of innovations: how goal attainment scaling programs fare over time. Community Mental Health Journal, 16, 130-143.

\title{
Behavioural and cognitive psychotherapy experience and supervision: a possible model of delivery
}

\author{
LYNNE M. DRUmmond, Consultant Psychiatrist and Senior Lecturer in Behavioural \\ Psychotherapy, St George's Hospital Medical School, Cranmer Terrace, \\ London SW17 0RE
}

In 1987 and 1989 surveys of junior doctors' experience of behavioural and cognitive psychotherapy in the South West Thames Region were published (Drummond \& Bhat, 1987; Drummond \& Bhat, 1989). These surveys demonstrated that a marked increase in the numbers of trainees gaining cognitive and behavioural experience was shown following monthly visits by the peripatetic Senior Lecturer in Behavioural Psychotherapy. The number of trainees gaining experience of treating at least one case under supervision increased from $17 \%$ to $42 \%$ over this period. However, this still fell short of the College recommendations that every trainee should receive this experience and treat at least two cases under supervision.

\section{Working party}

In 1987 a working party was convened in the South West Thames Region to ascertain the educational goals, content and processes for the learning of communication skills and psychotherapy by doctors working in the South West Thames Region who were training in psychiatry and preparing for entry into higher specialist training. This working party produced a document (Crisp et al, 1987) which set out educational goals and objectives as well as content and process for communication skills, dynamic and behavioural cognitive psychotherapies. The goals of behavioural psychotherapy training were set out as:

1. the ability to formulate the presenting problem in behavioural terms

2. the ability to assess a patient's suitability for treatment

3. the ability to design appropriate treatment programmes

4. the ability to implement the treatment

5. the ability to evaluate the outcome of treatment. 
The objectives were set out as:

1. consider complex or multiple problems and analyse them into more readily comprehensive components

2. enable realistic targets for each stage of treatment to be designed which do not overwhelm patients, relatives and staff or lead to feelings of therapeutic hopelessness

3. recognise when a problem is likely to be responsive to behavioural treatment

4. recognise the limitations of behavioural techniques

5. understand the various theories of behaviour, learning and cognition derived from experimental psychology which have relevance to human experience and responsiveness

6. utilise the above knowledge, recognising the psychological facts in clinical practice which may be involved in the development or maintenance of a patient's psychiatric or medical condition or problematic behaviour

7. be aware of the relevant research in the behavioural sciences

8. use the above knowledge in combination with the direct observation and assessment of a patient to produce and execute a realistic acceptable treatment package for the patient

9. be sensitive to the discomfort which patients may experience and respond to their visual cues and patients' reports of these experiences with appropriate empathy and encouragement

10. as a result of this awareness be prepared for flexibility within treatment sessions thereby allowing realistic and optimum treatment programmes to take place, taking into account the appropriate pace of treatment for an individual patient

11. understand the importance of objective measurement in assessment and monitoring of the efficacy of treatment

12. be able to design and apply relevant measures for a patient's problem

13. use each therapeutic account at its own single case experiment thereby avoiding any ineffective prolonged treatment

The process by which such treatment was to be delivered was debated and it was decided that in order to acquire the knowledge, skills and attitudes necessary for the goals and objectives, trainees required direct experience in treating suitable patients with behavioural and cognitive methods. During their training the trainees should, at least, have experience in the following:

1. exposure in vivo

2. self-imposed response prevention

3. operant programmes

4. sexual skills training

In addition, experience in the following would be an advantage:

1. imaginal exposure

2. social skills training
3. covert sensitisation and management of appetitive disorders

4. cognitive therapy of depression

In order to receive this experience during the three year training period the trainees should have completed behavioural treatment under supervision of five patients from each sub-section of Section $A$ and preferably one from Section B.

\section{Section A}

Obsessive compulsive disorder

Specific or social phobic

Agoraphobic

Sexual dysfunction

Social maladjustment, personality or behavioural disturbance.

\section{Section B}

Sexual deviation

Marital disharmony

Pathological grief

Obesity

Generalised anxiety

Depression.

It was decided that, to understand and apply behavioural techniques as well as meeting the needs of the MRCPsych. Part II Membership Exam, the trainee needed to attend a series of lectures which would introduce him or her to the scientific basis of behavioural psychotherapy. Thereafter further academic knowledge could be developed within the clinical supervision sessions and through further reading. A series of lectures were to be delivered to trainees on the Regional MRCPsych Part I Course as well as on the Regional MRCPsych Part II Course. These would cover the background of behavioural psychotherapy, relationship to learning theory and recent developments in academic psychology, research into its application for specific groups of patients and details of its practical application in treatment sessions.

In addition to the introductory lectures all of the teaching hospitals in the region have their own academic programmes which include behavioural topics and aim to keep trainees informed of recent research and developments in the area.

Trainees were also expected to do some background reading in experimental psychology and learning theory as well as in practical application of clinical behaviour therapy. In addition they would aim to keep up to date with literature by reading relevant journals.

The document and the recommendations of the working parties were accepted by the South West Thames British Postgraduate Medical Federation 
Psychiatric Sub-committee and it was agreed that adherence to these goals should be an integral part of all registrars' training throughout the South West Thames Region. Satisfactory attainment of the goals were agreed to be necessary before a registrar was deemed ready to sit for the MRCPsych. Final Examination. These expectations were written into the job contract for all trainees joining the region.

\section{Process}

The South West Thames Region at the time of writing has 43 SHOs and 76 registrars. Clearly it would be impossible for one peripatetic senior lecturer to offer high quality supervision for each trainee to gain behavioural psychotherapy experience and treat a minimum of five cases. For this reason a Behavioural Psychotherapy Supervisor was appointed for each of the districts in the South West Thames Region in 1989. These psychotherapy supervisors were all consultant psychiatrists. It was estimated that one session a week would be required to administer their programme, offer supervision and teaching to trainees on a weekly to fortnightly basis and to evaluate the results of such teaching. As no extra funding was available for this purpose, consultant colleagues throughout the region were asked to undertake some of the behavioural psychotherapy supervisors' workload to enable the latter to devote one session a week to their teaching. Although we did not expect our request to ask already busy consultant colleagues to undertake more work would be popular, the reality of there being 139 consultant psychiatrists in the region and only 13 behavioural psychotherapy supervisors has meant that this has not been a problem in practice.

An initial difficulty was finding consultant psychiatrists with relevant expertise in behavioural and cognitive psychotherapy. Although a refresher course in behavioural and cognitive psychotherapy was conducted by the senior lecturer at the outset of this system this is clearly inadequate if the consultant had little prior knowledge of the methods. Individual training of a consultant by the senior lecturer would clearly not have been a cost efficient exercise when other courses are available. In practice most districts do have a consultant with considerable behavioural and cognitive skills, many having completed the Association of University Teachers of Psychiatry Course in Behavioural Psychotherapy run at the Institute of Psychiatry in London. In those districts where no consultant had this experience we have found the Mental Health Units have generally been willing to fund a consultant to attend the AUTP Course at the Institute of Psychiatry. This willingness has, no doubt, been helped by the steadfast approach of the South West Thames British Postgraduate Medical Federation Psychiatric Sub-committee insisting that such a consultant must be in post in a district if the SHO and registrar posts in that district are to be considered suitable for incorporation into the St George's Teaching Hospital rotational training schemes.

The weekly to fortnightly supervision sessions are run by the Behavioural Psychotherapy Supervisor and backed up by the Peripatetic Senior Lecturer. The sessions from the senior lecturer have generally taken the form of more difficult cases being presented for discussion.

\section{Outcome}

Full evaluation of outcome is not currently available. However, it does appear that all psychiatric trainees in the South West Thames Region are now gaining at least some supervised behavioural psychotherapy experience during their training.

\section{Conclusion}

A description has been given of one scheme to ensure all psychiatric trainees gain some experience in behavioural and cognitive psychotherapy. It has been found that considerable expertise in these matters can be found among consultant psychiatrists although many had, prior to the reorganisation of the teaching, been 'hiding their light under a bushel'. The delivery of behavioural and cognitive psychotherapy at district level is remarkably cost efficient with only one session a week of consultant time being required. This time input has been backed up by the Peripatetic Senior Lecturer who also has input in terms of organisation as well as providing additional academic and supervisory sessions.

We believe that this system is much more efficient than the practice used in many regions where psychologists are expected to deliver the bulk of cognitive and behavioural training to the psychiatric trainees. Although it is felt to be acceptable by the College in some cases when a consultant is not available to delegate the training of junior doctors in behavioural psychotherapy to psychologists (Royal College of Psychiatrists, 1986) it does have many disadvantages. Firstly, although there are a number of psychologists and other paramedical therapists who express some interest and willingness to train junior doctors in the Region (Heavey et al, 1986), in practice this rarely happened and few trainees received any supervision or training from this source (Drummond and Bhat, 1987). Psychologists and paramedical personnel often have heavy clinical commitments as well 
as the expectation of training their own and various other professional groups. This may result in the training of junior doctors not receiving the priority it requires. Junior doctors with busy clinical jobs often do not attend all teaching sessions and it requires a highly motivated supervisor who can liaise with other senior medical staff to increase attendance. It is also often easier for a senior member of the medical staff to emphasise the importance of this training to other senior medical colleagues. Trainees themselves are also likely to attach more importance to training offered by a senior member of their own profession and are likely to view such training as being more relevant to their own needs. Moreover, there are many paramedical professionals who claim behavioural and cognitive expertise but who, in practice, are familiar with only a small range of patients. Catchment area psychiatrists, however, see many wide-ranging difficult and chronic patients and the application of behavioural and cognitive techniques to these groups requires a different repertoire of skills than a more limited clientele.

\section{References}

Crisp, A. H., Burns, T., Drummond, L. M., et al (1987) The learning of communication skills and psychotherapy by doctors training in psychiatry and preparing for entry into higher specialist training, and working in the South West Thames R.H.A. Published by The Professiorial Unit, Department of Mental Health Sciences, St George's Hospital Medical School, Tooting, London SW17 ORE.

Drummond, L. M. \& BhAT, A. V. (1987) A regional survey of the training of junior psychiatrists in behavioural psychotherapy. Bulletin of the Royal College of Psychiatrists, 11, 126-128.

- (1989) A two year update of a regional survey of the training of junior psychiatrists in behavioural psychotherapy. Psychiatric Bulletin, 13, 296-298.

Heavey, A., Crisp, A. H. \& Lieberman, S. (1986) A Survey of Psychotherapy Training Resources and Activity in the South West Thames Regional Health Authority. Document held by the Psychiatric Sub-committee of the South West Thames British postgraduate Medical Federation.

The Royal College of Psychiatrists (1986) Guidelines for the training of general psychiatrists in psychotherapy. Bulletin of the Royal College of Psychiatrists, 10, 286-289.

\title{
Trainees' forum
}

\section{Patients repeatedly admitted to psychiatric wards}

\author{
Mavis Evans, Senior Registrar; David Rice, SHO; and Christina Routh, Registrar; \\ Directorate of Psychiatry, Wirral Hospital NHS Trust, Bebington, Wirral, \\ Merseyside L63 4JY
}

Many admissions to general adult psychiatric beds occur as emergencies, either due to social crisis or relapse of psychiatric illness. Other admissions are planned to arrest or reverse deterioration in mental state. Length of stay varies from days to months depending on reason for admission and adequacy of support in the community. Rapid readmission or failed discharge, defined as readmission within three months, has been linked with multiple previous admissions and personality factors (Jones, 1991).

All units have a few "revolving door" patients who are frequently readmitted although their illnesses and social circumstances do not appear to differ markedly from others who can live successfully in the community for longer periods. We defined such patients as those admitted six or more times within 12 months.

The aim of this study was to identify factors which would differentiate those patients admitted frequently from those remaining in the community for longer periods.

\section{The study}

A retrospective review of admissions over 12 months was carried out on two acute psychiatric wards in a district general hospital (DGH), a 10 bed high dependency ward and a 25 bed medium dependency ward. Patients are transferred between these wards as necessary, allowing disturbed behaviour to be controlled with the minimum of sedation. The DGH serves a catchment population of approximately 360,000 , with 90 adult medium dependency beds and 20 adult high dependency beds. The catchment area is mixed rural and urban with a level of unemployment higher than the national average.

Case-note review was undertaken on the "revolving door" patients. As these patients had a history of frequent discharge against medical advice (DAMA), a further review of all recorded DAMA on the wards over the same period was carried out, with case-note review of all patients involved. 